\title{
An Efficient Power Delay Product of ZigBee Transmitter Using Verilog HDL
}

\author{
Raja Prakasha Rao Pasala ${ }^{1}$ and Rajendra Naik Bhukya ${ }^{2}$ \\ ${ }^{I}$ Ph.D Research Scholar, Department Of Electronics And Communication Engineering, Shri JJT University, \\ Rajasthan, India \\ ${ }^{2}$ Head, Department Of Electronics And Communication Engineering, University College Of Engineering, \\ Osmania University, Hyderabad, Telangana State, India
}

\begin{abstract}
In this paper, we have focused on the ZigBee wireless technology, various topologies of ZigBee technology and design of ZigBee Transmitter chip using Verilog HDL programming language. Introduction of wireless interface devices increased the demand for low-data-rate and short-range wireless networking systems. Thus, the new communication protocol ZigBee was developed for wireless transmission. This paper presents the ZigBee transmitter for MAC layer according to the IEEE 802.15.4 standard. Design results prove that the area and power has been reduced with the proposed technique. In this paper we have also presented the power delay product of a ZigBee transmitter. We have obtained the 99\% of errors detected in proposed work with the use of CRC algorithm. The proposed design has been synthesized in Xilinx ISE software.
\end{abstract}

Keywords: ZigBee, MAC layer, Verilog HDL, CRC.

\section{Introduction}

Wireless personal area network (WPAN) and a wireless local area network (WLAN) technologies are growing fast with the new emerging standards being developed. For short range communications mostly Bluetooth was widely used. Nowadays, ZigBee is used as an alternative to Bluetooth for the device applications which consumes less power. The Bluetooth standard format is a specification for WPAN. Some products which are based on the Bluetooth standards can also be used for longer distances. Actually Bluetooth uses a short range radio link which operates with a $2.4 \mathrm{GHz}$ similar to WLAN. Thus the radio links which are used in Bluetooth are based on frequency hop spread spectrum. ZigBee Alliance is the one who developed the ZigBee standards which is now utilized by software companies to manufacturing industries. In the year 2002, the ZigBee alliance was formed and opened for all individuals to join. IEEE 802.15.4 is standard adopted as a ZigBee standard and uses protocol frame structure based on this IEEE standard as well.

Mostly ZigBee standard was developed to serve the devices with low data rate wireless networks with low power and low cost implementation. The ZigBee standard achieved low implementation cost by modifying the communication protocol. The minimum needs to meet the ZigBee and IEEE 802.15.4 specifications are matched when to other standards which improved in complexity reduction and reduced cost for implementation of ZigBee compliant transceivers. ZigBee wireless networking protocol layers are shown in Fig.1. Based on OSI basic reference model ZigBee protocol layers are formed. The two bottom layers are defined by IEEE 802.15.4 standard and this standard was released and developed by IEEE 802 standards committee in year 2003. The PHY and MAC layer specifications are defined by IEEE 802.15.4 for wireless networking.

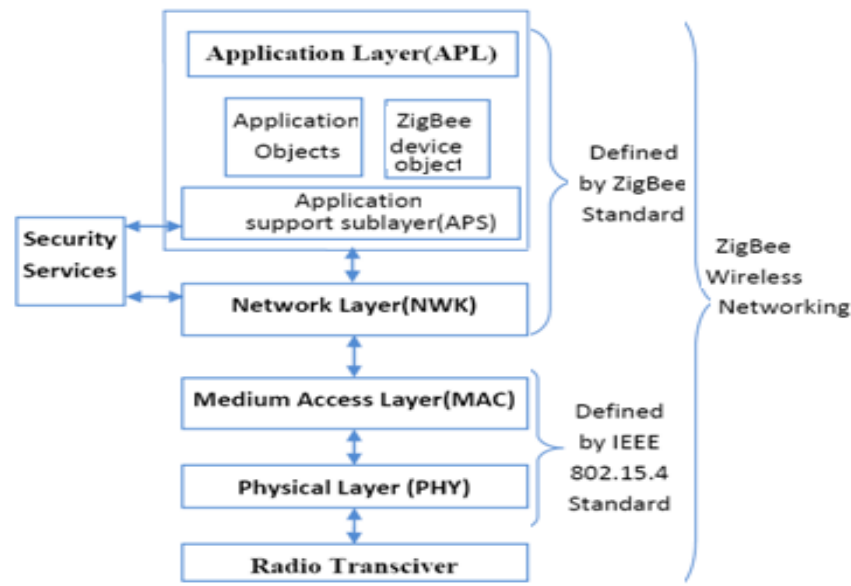

Figure 1: ZigBee wireless networking protocol layer 
The $915 \mathrm{MHz}$ and $2.4 \mathrm{GHz}$ bands are part of frequency bands of industry, scientific and medical (ISM) bands. Especially in North America a $915 \mathrm{MHz}$ frequency band is use, whereas the $2.4 \mathrm{GHz}$ band is used worldwide. The Table-1 given below provides additional details regarding the ways in which these three frequency bands are used in IEEE 802.15.4 standard [3]. The standard rule of IEEE 802.15.4 requires to be supporting the $915 \mathrm{MHz}$ band if it supports the $868 \mathrm{MHz}$ band for transceiver. Therefore, the two bands are bundled together to operate in $868 / 915 \mathrm{MHz}$ frequency bands. IEEE 802.15.4 has a one mandatory rule and two optional specifications for the $868 / 915 \mathrm{MHz}$ bands.

The mandatory requirements yields to low data rate but can be implemented simpler. If a user selects to implement the operation in optional modes, still IEEE 802.15.4 requires that it adopt the mandatory low data rate operation in the $868 / 915 \mathrm{MHz}$ bands as well. Thus the transceiver must also design to switch dynamically between mandatory and optional modes of operation bands. A $4 \mathrm{GHz}$ transceiver can support $868 / 915 \mathrm{MHz}$ bands, but IEEE 802.15 .4 is not recommended. The $2.4 \mathrm{GHz}$ band contains total of 16 channels. Throughout the world $2.4 \mathrm{GHz}$ ISM band is accepted which has maximum data rate and number of channels. Due to this it is more popular for $2.4 \mathrm{GHz}$ transceiver development by many manufacturers. Many users mostly prefer for lower signal frequency bands because as the frequency is less there is less chances of signal penetrates over the walls. Therefore, some users may find the $868 / 915 \mathrm{MHz}$ band a better choice for their applications.

Table 1: Worldwide operating frequency range of ZigBee

\begin{tabular}{|c|c|}
\hline Geography & $\begin{array}{c}\text { Standard Regulatory } \\
\text { frequency Range }\end{array}$ \\
\hline China & $784 \mathrm{MHz}$ \\
\hline Europe & $868 \mathrm{MHz}$ \\
\hline USA and Australia & $915 \mathrm{MHz}$ \\
Other Nations & $2.4 \mathrm{GHz}$ \\
\hline
\end{tabular}

\section{Topologies Of ZigBee}

IEEE 802.15.4 networks have been divided into two topologies; those are star and peer-to-peer. A cluster tree structure is defined as a variation of peer-to-peer topology. Compared to the other topologies, only star topology has the most configuration structure. The Personal Area Network coordinator node always sits in the center of the network

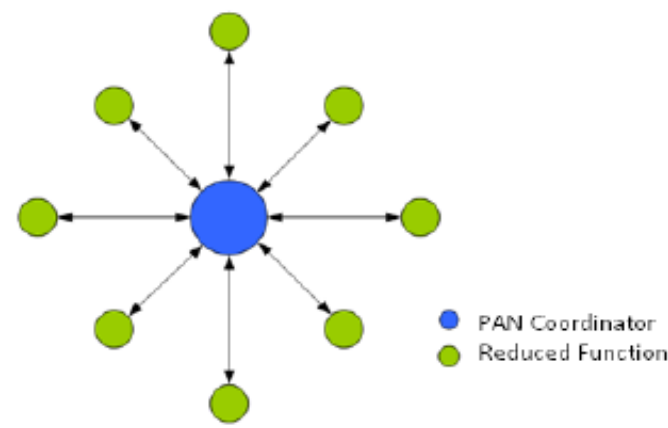

Figure.2: Star Network Topology

All the other nodes need to communicate via the PAN coordinator node. The PAN Coordinator node has been used in network to send the messages to other nodes. Between any two nodes direct communication is not allowed as shown in the Figure 3. The special case of a peer to peer topology is a cluster tree topology. This was based on RFDs only which can communicate with FFDs. Majority of FFDs devices are present in cluster trees as shown in Figure.4.

The ZigBee Alliance has been formed in the year 2002 as a nonprofit organization which is open to all who wants to join. The ZigBee standard uses the PHY layer and MAC layer protocol based up on the IEEE 802.15.4. Hence, a ZigBee device is compliant with the IEEE 802.15.4 standard a well. The Physical Layer supports three frequency bands such as $2.45 \mathrm{GHz}$ band with 16 channels, an $868 \mathrm{MHz}$ band with 1 channel and a $915 \mathrm{MHz}$ band with 10 channels. However main focus of this research paper is on $2.45 \mathrm{GHz}$ band of 
frequencies which is being used throughout the world, with a data rate of $250 \mathrm{kbps}$. The MAC layer defines two types of nodes: Reduced

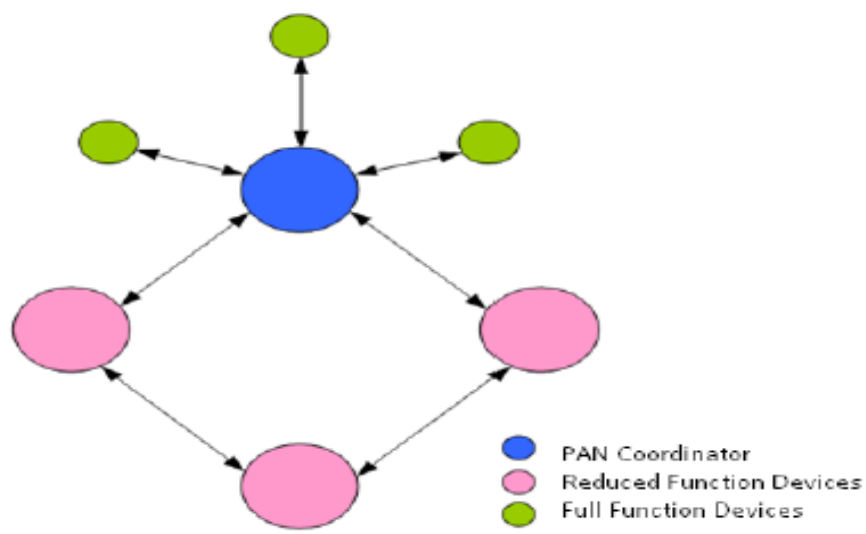

Figure.3: Peer-to-Peer Topology

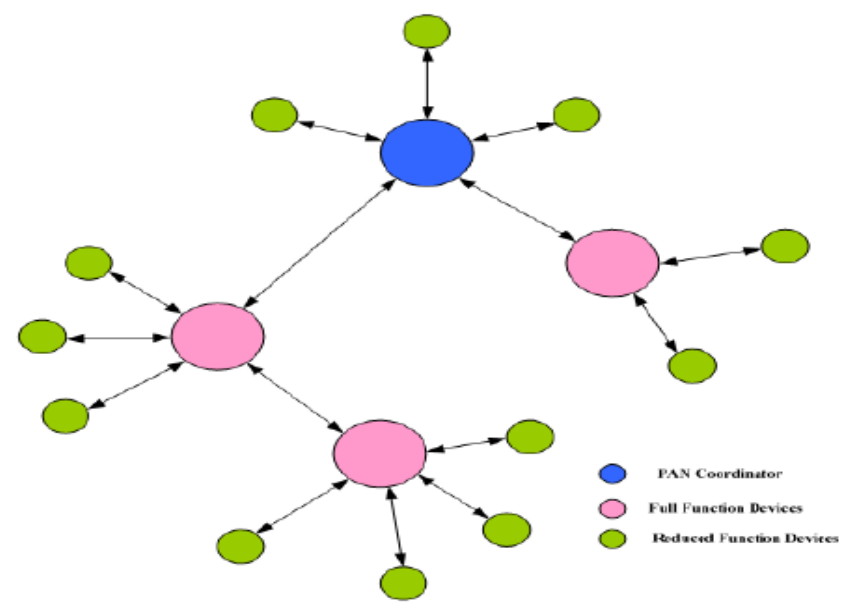

Figure.4: Cluster Tree Topology

Function Devices (RFDs) and Full Function Devices (FFDs). RFDs can only act as end-devices and are equipped with sensors or actuators like transducers, light switches and lamps. They may only interact with a single FFD [7, 8]. FFDs are equipped with a full set of MAC layer functions, which enables them to act as a network coordinator or a network end-device [1,4]. Two main type of ZigBee networking topologies are star and peer-to-peer. In the star topology, every device in the network can communicate only with the personal area network (PAN) coordinator. A FFD takes up a role as PAN coordinator; the other nodes can be RFDs or FFDs. In Peer-to-Peer Network topology, each device can communicate directly with any other device if the devices are close enough together to establish a successful communication link. In this topology any Full Function Device and play the role of the PAN Coordinator [8].

The IEEE 802.15.4 defines four MAC frame structures: beacon, data, acknowledgment, and MAC command frames. A coordinator uses a beacon frame to transmit beacons. The function of beacons is to synchronize the clock of all the devices within the same network. The data frame is used to transmit data.

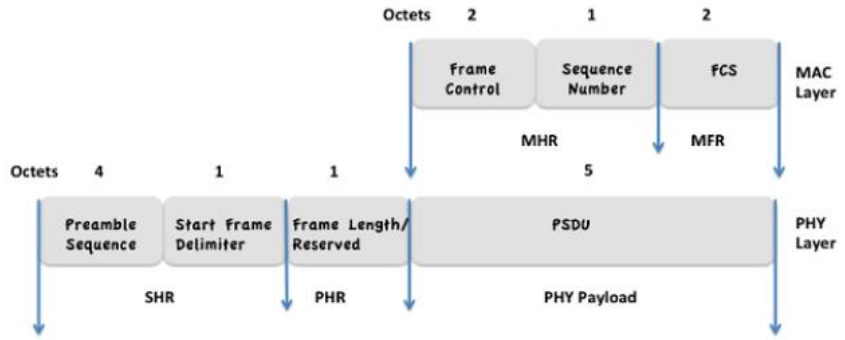

Figure.5 The acknowledgment frame [24] 
Meanwhile, the acknowledgement frame is used to confirm successful frame reception $[1,8]$. The MAC commands are transmitted using a MAC command frame. Based up on IEEE 802.15.4 standards the ZigBee digital transmitter has been designed for an Acknowledgment frame. This is the simplest MAC frame format and does not carry any MAC payload.MAC frame includes MAC header (MHR) and the MAC footer (MFR).The MHR is formed by the frame control field and direct sequence number. The MFR consists of 16 bit frame check sequence (FCS). Both MHR and MFR are also known as PHY service data unit (PSDU), which becomes the PHY payload. The PHY payload includes preamble sequence, synchronization header, start of frame delimiter (SFD) and PHY header (PHR). PHY protocol data unit (PPDU) is formed by SHR, PHR and PHY payload. Length of preamble sequence field is 4 octets. As for SFD, the length is 1 octet. The PHR also contains 1 octet. This is followed by the MAC Header and the MAC Footer with 3 octets and 2 octets, respectively. Therefore the acknowledgement frame contains 11 octets in total.

Major applications of ZigBee focus on sensor and automatic control, such as healthcare, industrial control, home automation, remote control, and monitoring systems [8].For example in health care of a patient at home wearing a ZigBee device can be monitored by his physician continuously. The information of patient heart rate and blood pressure is transmitted over the IEEE 802.15.4 network to a personal computer that the physician or nurse uses to monitor the patient. This system can help hospitals to improve caring of patients and avoid overcrowding of hospitals by enabling them to monitor patients at home. ZigBee mess networking can help at industrial level in areas such as energy management, light control, process control and asset management. The ZigBee can be used in home automation for example it can be used for power meter reading, security, light control, multi zone heating, ventilation and air-conditioning (HVAC) systems. ZigBee also can be used in wireless remote control for communication with televisions, DVDs, and other entertainment devices via infrared signals. For monitoring systems, ZigBee can be used to monitor hotel guest room access and fire extinguishers $[1,2]$. The transmission range for ZigBee devices is $10-100 \mathrm{~m}$, based on the environment [5].The battery lifetime is up to 2-year [6].For large designs schematic is not appropriate where more logic functionality is involved. Shuaib et al.[8] developed and simulated the transmitter design using Matlab. Unfortunately, this design has not yet been implemented. In contrast, Meng et al. has designed, implemented and tested the ZigBee receiver with a commercially available off-the-shelf ZigBee transceiver.

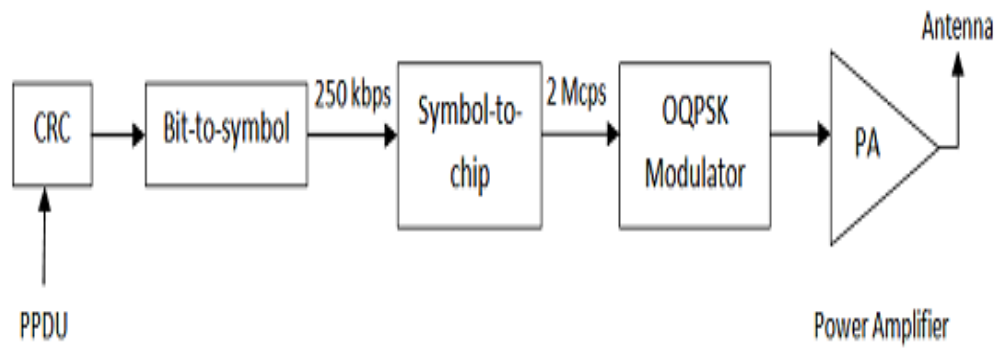

Figure.6: Detailed architecture of the proposed digital transmitter.

\section{Transmitter Architecture}

For 2.4 GHz-band ZigBee applications, sixteen channels are available with $5 \mathrm{MHz}$ ample channel spacing. IEEE 802.15.4 standards work on Direct Sequence Spread Sprectrum (DSSS) which uses a digital spreading function which represents pseudo-ramdom noicse (PN) chip sequence which is shown in the Table 1. The acknowledgement frame contains octets of PPDU. Figure 6 shows the architecture of the proposed ZigBee digital transmitter. Binary data from the PPDU packet are inserted into the cyclic redundancy check (CRC) block to detect errors during transmission. CRC is the most preferred method of encoding because it provides very efficient protection against commonly occurring burst errors [2], and is easily implemented [3].

CRC's can detect all one bits and two bits errors as well as all odd number of bits in error [4, 8]. Since $\mathrm{CRC}$ is a technique for detecting errors, but not for making corrections when errors are detected, the whole packet data will be retransmitted if error occurs [5]. For any ZigBee Standard CRC algorithm involves a division of the transmitted packet of data by a constant called the generator polynomial $[1,8]$. In this paper, the CRC block contains the SHR, PHR and PHY payload. The FCS mechanism works on 16-bit CRC in any PHY payload in order to detect errors [1,3]. The FCS is calculated over the MHR and MFR payload parts of the frame using the algorithm in figure 6.

Then each of four bits of PPDU octet is mapped onto one data symbol. The mapping takes place in a bit-to-symbol block. The 4 least significant bits (LSBs) (b0, b1, b2, b3) of each octet is mapped into one data symbol and the 4 most significant bits (MSBs) (b4, b5, b6, b7) of each octet is mapped into the next data 
symbol. Every PPDU octet is processed through the bit-to-symbol block in a sequential order, begins with Preamble field and ends with the last octet of the PSDU.

A. Cyclic redundancy check

Error detection is a process to generate the redundant bits using the CRC polynomial with respect to data transmission and identifies the error occurred by comparing the CRC generated with the receiving CRC. Error-detection techniques only detects whether an occurred in the transmitted but will not correct any errors nor identifies the error position. The purpose of error detection is not to prevent errors from occurring but to prevent undetected errors from occurring. The most common error detection techniques are redundant bit check such as vertical redundancy checking, checksum, longitudinal redundancy checking, and cyclic redundancy checking.

\section{B. CRC polynomial}

The most reliable redundancy checking algorithm for detecting errore is a convolution coding scheme which is called as cyclic redundancy check algorithm (CRC).With CRC, approximately $99.999 \%$ of all transmission errors are detected. In CRC-16, 16 bits are used for the block check sequence. Here, the entire data stream is treated as a long continuous binary number. The Block Check Sequence (BCS) is transmitted separately from the message and CRC is considered a systematic code. Cyclic block codes are often written as $(\mathrm{n}, \mathrm{k})$ cyclic codes where $\mathrm{n}=$ bit length of transmission and $\mathrm{k}=$ bit length of message. Therefore, the length of the Block Check Character (BCC) in bits is

$$
\mathrm{BCC}=\mathrm{n}-\mathrm{k}
$$

A CRC-16 $\mathrm{BCC}$ is the process of binary division to obtain the remainder. $\mathrm{P}(\mathrm{x})$ is the generator polynomial is used to divide the message polynomial $\mathrm{G}(\mathrm{x})$ to obtain the remainder and append to the $\mathrm{BCS}$ message. The generator polynomial must be a prime number. With CRC algorithm we cannot achieve the standard arithmetic division. In the proposed system the division logic is obtained by using modulo- 2 division and the remainder is obtained by XOR operation. Whereas in the receiver data stream including the CRC code is divided by the same generating function $\mathrm{P}(\mathrm{x})$. The remainder will be zero if no transmission errors are present in the received data.

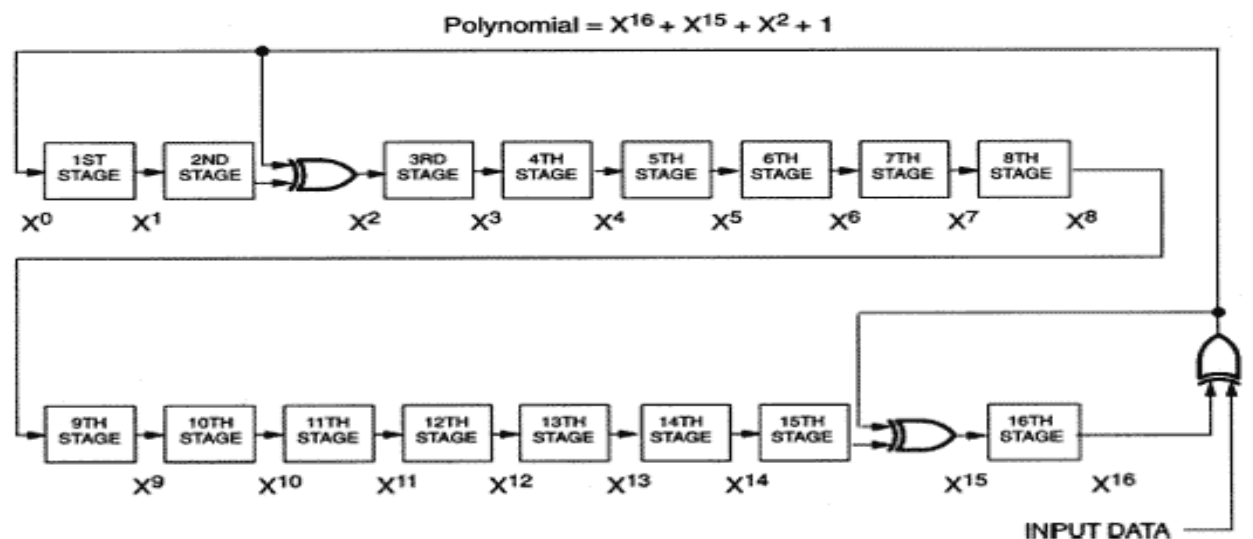

Figure.4 CRC-16 Generating circuit

Mathematically, a CRC can be expressed as

$$
\begin{aligned}
& G(x) \\
& -----=Q(x)+R(x)
\end{aligned}
$$

Where,

$\mathrm{G}(\mathrm{x})=$ message polynomial,

$\mathrm{P}(\mathrm{x})=$ generator polynomial,

$\mathrm{Q}(\mathrm{x})=$ quotient,

$\mathrm{R}(\mathrm{x})=$ remainder,

The generator polynomial for CRC-16 is

$\mathrm{P}(\mathrm{x})=\mathrm{x} 16+\mathrm{x} 15+\mathrm{x} 2+\mathrm{x} 0$ 


\section{Proposed method algorithm:}

\section{Design Methodology}

The design algorithm defines the frame structure generated by ZigBee MAC layer as follows in figure 6. The frame of Generic MAC layer has got frame control field of 2 octets. It carries useful information such as frame type, source and destination addressing modes. The frame is beacon frame, date frame, ACK of date, MAC command frame etc., is being specified by the Frame type. The same is outlined 'Frame type subfield' table below with 3 bits. The Sequence number describes the frame sequence order. Destination address is sent first to indicate the target device which is ready to receive the data. Source address is sent after to indicate from which device data is received to the target. The outgoing frame is whether security enabled or not is indicated by Security control Field. The Actual data is sent on a payload field. At last the frame check sequence is sent to tell the receiver whether the data is received correctly at the destination or it may collided with unknown data. The receiver also calculates the frame check sequence according to the same polynomial used by the transmitter to calculate the frame check sequence.

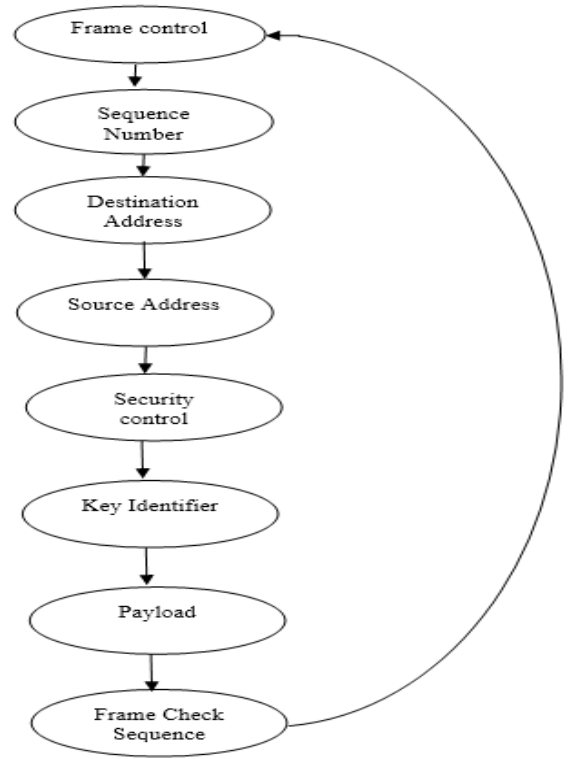

Figure 5: Algorithm used for ZigBee MAC transmitter design

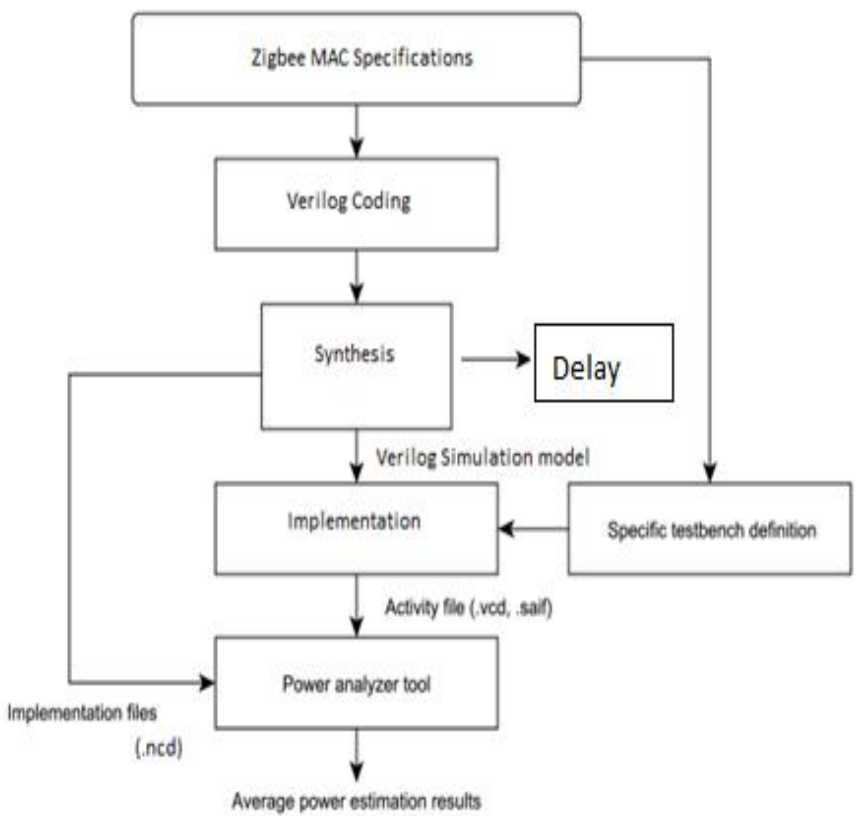

Figure 6: Algorithm used to obtain the power and delay results in Xilinx ISE tool 


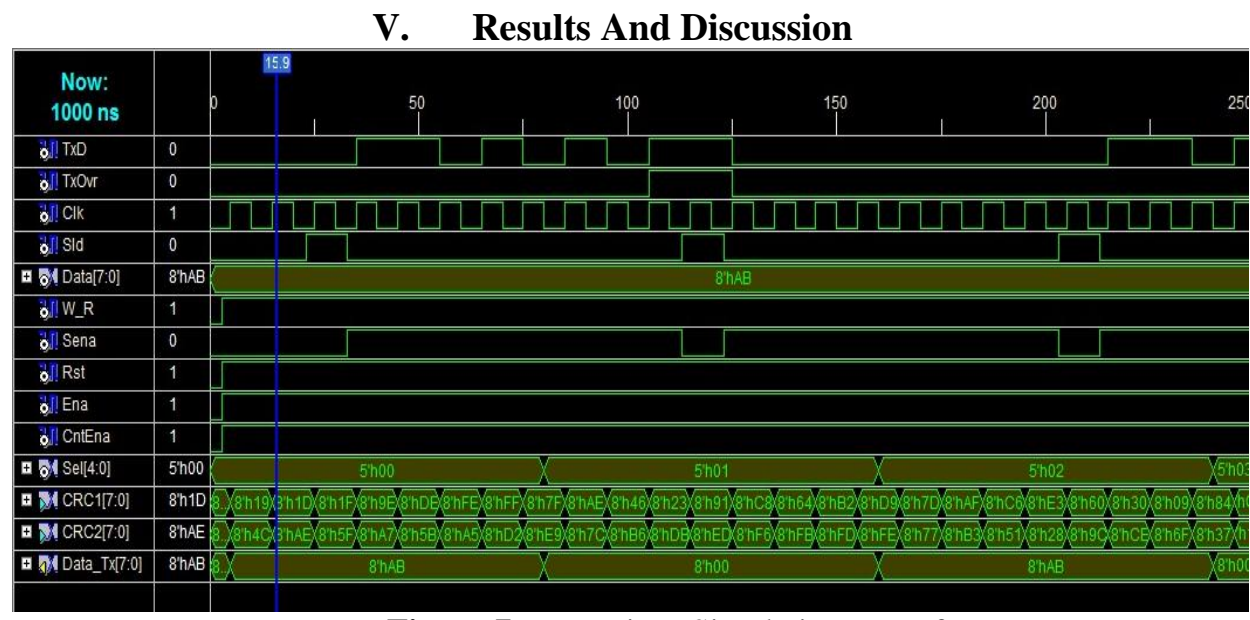

Figure.7 Transmitter Simulation Waveform

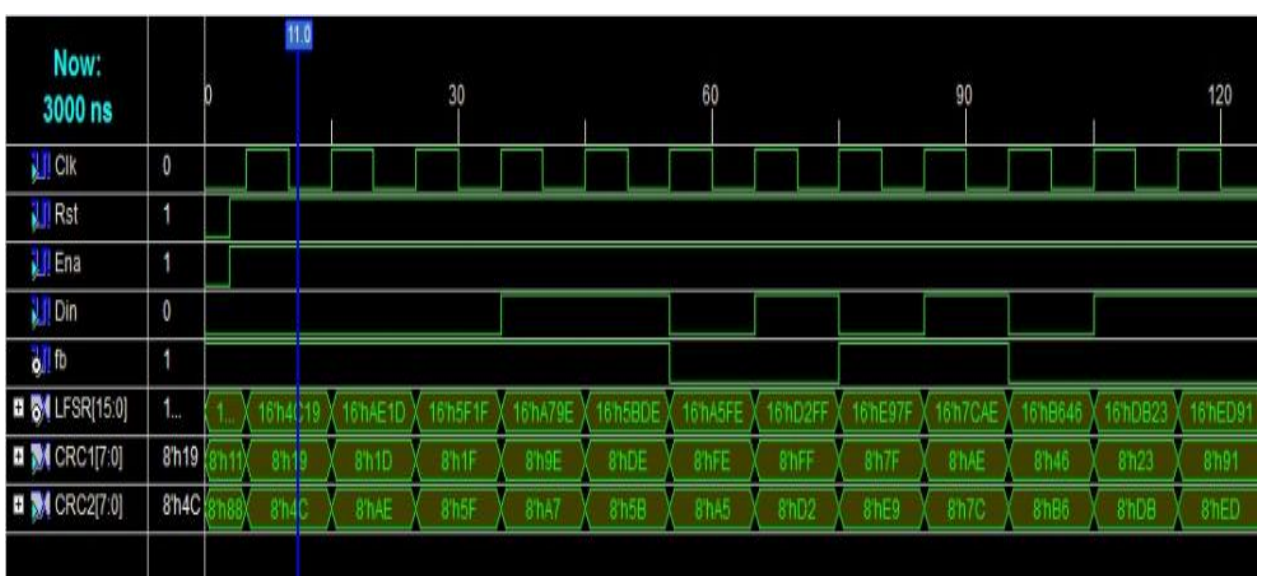

Figure.8 CRC Simulation Waveform

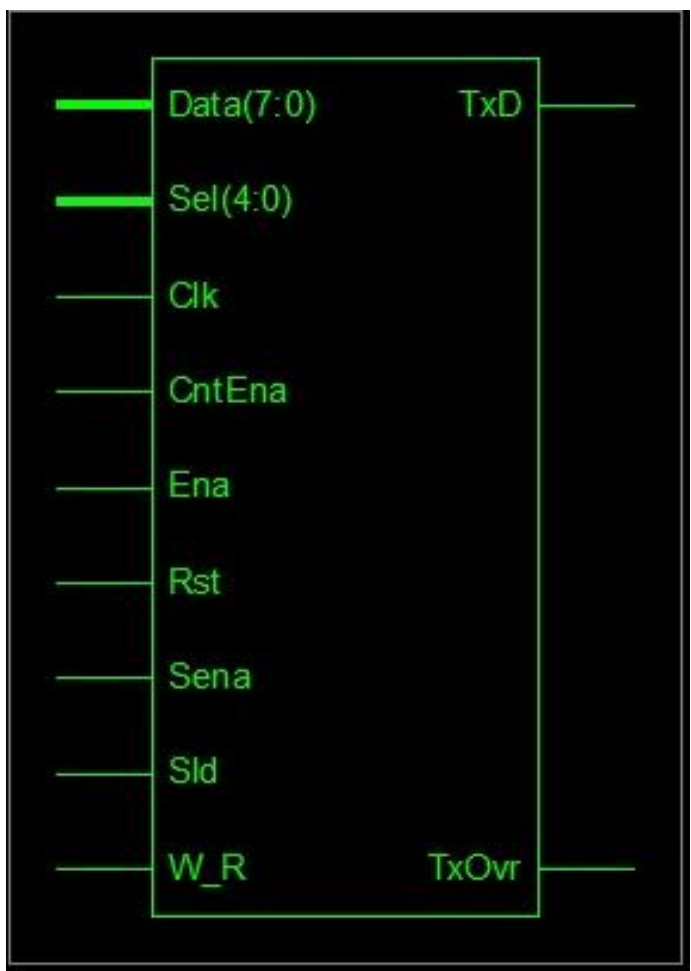

Figure.9 RTL schematic of ZigBee transmitter 


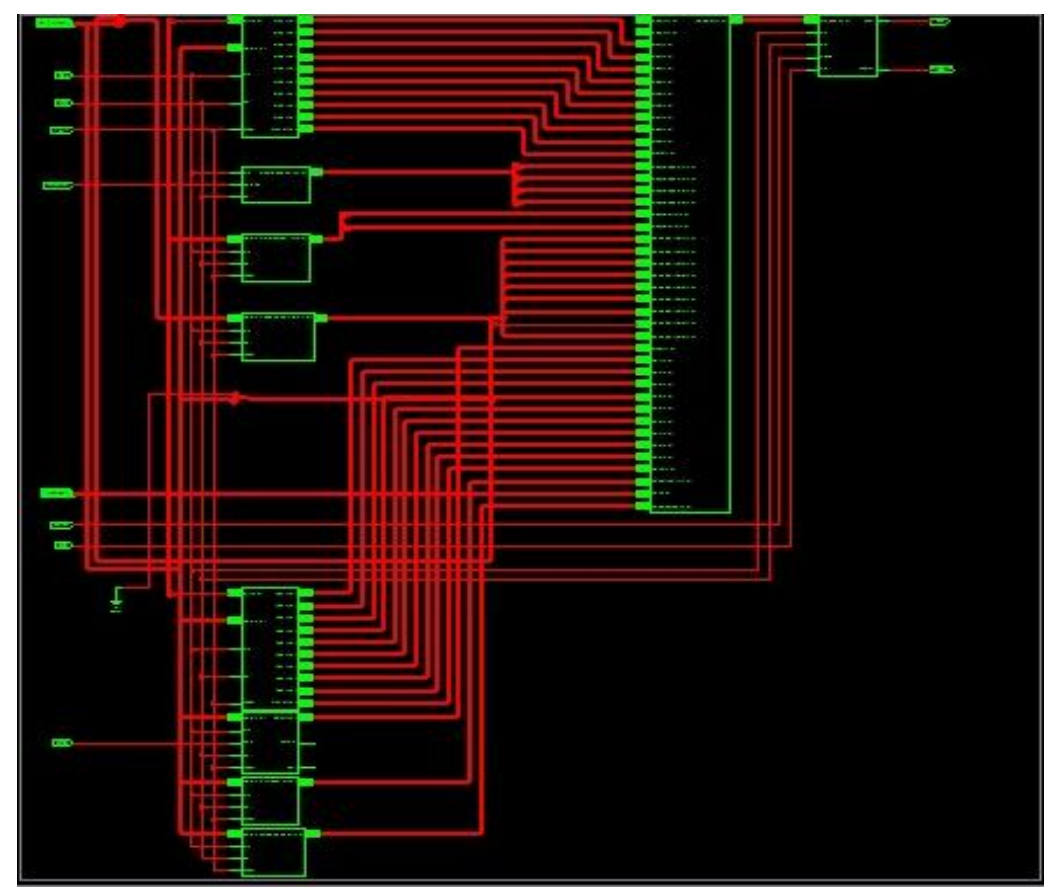

Figure.10 RTL schematic of ZigBee transmitter

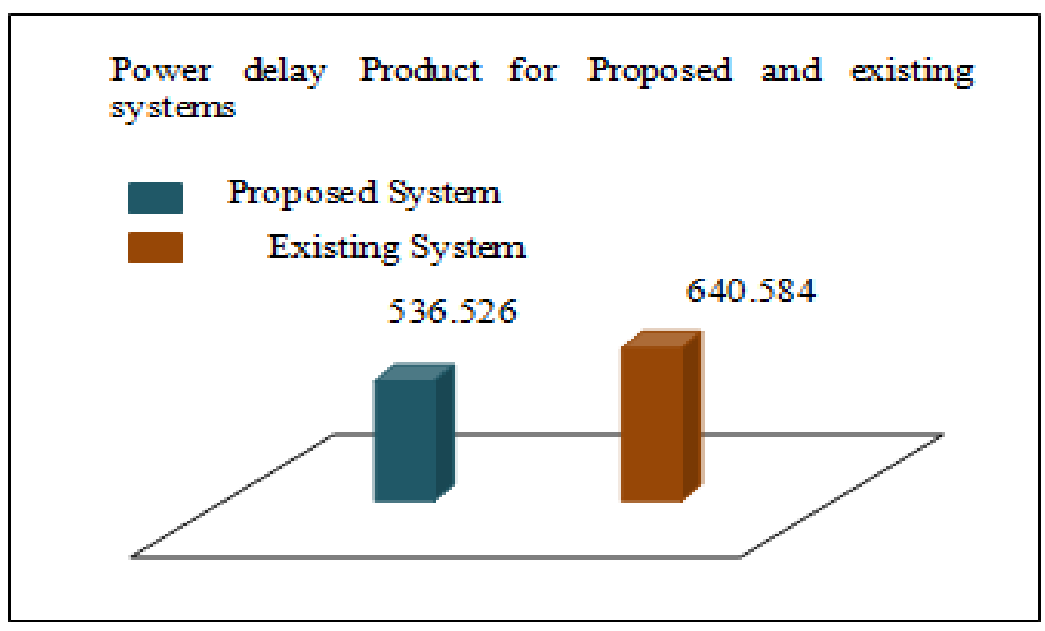

Fig.11 Pictogram representation of power delay product

\section{Conclusion}

This paper shows the Verilog based design of digital transmitter for $2.4 \mathrm{GHz}$ band ZigBee applications. The behavior of CRC and Bit-to-symbol were characterized using Verilog. From the discussion, so far, part of the ZigBee transmitter is alone is characterized and synthesized. The synthesis is done by using XILINX ISE and targeted for SPARTAN 3E FPGA.The results of Synthesis for the proposed method shows reduced number of slices and LUTs when compared to existing method.. Hence the proposed method proves the more efficient in power delay product.

\section{Acknowledgements}

I would also like to acknowledge the enthusiastic co-supervision Dr. Rajendra Naik, Head, Department of ECE, University College of Engineering, Osmania Univeristy for his valuable guidance and support extended throughout the research. It is his co-operation and kindness, that I could complete my research paper. My grateful, appreciation for him, for providing Xilinx lab facility and other facilities during the research. 


\section{References}

[1]. Khalifa. O, Islam. MDR and Khan. S, "Cyclic redundancy encoder for error detection in communication channels", RF and Microwave Conference, 2004

[2]. Matloff .N, "Cyclic redundancy checking”, Department of Computer Science, University of California, 2001.

[3]. Rafidah Ahmad, Othman Sidek, Wan Md. Hafizi Wan Hassin, Shukri Korakkottil Kunhi Mohd, and Abdullah Sanusi Husain, "Verilog-Based design and implementation of Design Tansmitter for ZigBee Applications", International Journal of Emerging Sciences, 723-724, December 2011, ISSN: 2222-4254, (C) IJES.

[4]. Rahmani.E, "ZigBee/IEEE 802.15.4”, University of Tehran, 2005

[5]. Shuaib.K, Alnuaimi.M, Boulmalf.M, Jawhar.I, Sallabi.F and Lakas.A, "Performance evaluation of IEEE 802.15.4:Experimental and simulation results", Journal of Communications, 2, 4(2007): 29-37.

[6]. Sunil Shukla, Neil W.Bergmann, "Single bit error correction implementation in CRC-16 on FPGA", Proceedings of IEEE International Conference on Field-Programmable Technology 2004.

[7]. Ting Ting Meng, Chen Zhang, Peter Athanas, "An FPGABased ZigBee Receiver on the Harris Software Defined Radio SIP", SDR Forum Technical Conference, Denver, Colorado, 2007.

[8]. Wayne Tomasi, "Advanced Electronic Communication System", 6th Edition, Pearson Education.

[9]. ZigBee Alliance, available at: www.ZigBee.org. 